\title{
Generación y gestión de residuos sólidos ordinarios en la Universidad Nacional de Costa Rica: patrones cuantitativos y sociológicos
}

\author{
Zaidett Barrientos \\ Laboratorio de Ecología Urbana y Sistema de Estudios de Posgrado, Universidad Estatal a Distancia (UNED), Costa Rica; zbarrientos@uned.ac.cr
}

Recibido 12-IV-2010 Corregido 22-V-2010 Aceptado 24-V-2010

\begin{abstract}
Urban solid waste generation and management at the National University, Costa Rica: quantitative and sociological patterns. The National University (Costa Rica) established an environmental management program that includes a waste management project. This article evaluates the performance of the project during 2005, and also experimentally and quantitatively analyzes the process. The main obstacles were design faults in the storage structures, inadequate procedures, contradiction between plans and actions, confusing signage and rejection by the university staff from (based on conflicts of interest due to economic and labor interests, and from personal aesthetic views. That year a total of $53560 \mathrm{~kg}$ (mean: $6 \mathrm{~kg} /$ person) were processed. Paper and paperboard predominated ( $82 \%$ of waste), followed by glass $(8 \%)$, plastics $(4 \%)$ and metals (2\%) (organic waste was not considered). The Publications Office produced most waste was. There was a trend to an increase in "extramural" waste (residents, staff and students bringing waste from their homes). The correct disposition of waste improved after a change in the labeling of problematic bins (Paper 96-95\%, Non-recyclable $88-90 \%$. When Various was changed to Containers: plastic, glass and aluminum, correct disposition went from 51 to $75 \%$ ). The improvement in the classification of waste is probably the result of education and improvements in organization and labeling. In the middle of the period, waste sent to the landfill had a daily average of $606,7 \mathrm{~kg}$, but only $3,6 \%$ was recyclable material, mainly plastics and paper. The sites where the material was stored for shipment to the landfill had poor hygiene when cleaning when anti-vertebrates barriers made cleaning difficult. In designing this type of project, it is necessary to consider all participant groups to avoid conflicts over work roles and economic inputs. The benefit of this type of project should be viewed from a broader perspective, because depending on the conditions of each country, there may be no real economic income.
\end{abstract}

\section{KEY WORDS}

Implementation of recycling, ecocampus, environmental management, university wastes, urban solid waste management.

\section{RESUMEN}

La Universidad Nacional (Costa Rica) estableció un programa de gestión ambiental que incluye un proyecto de manejo de residuos ordinarios. Este artículo evalúa el desempeño del proyecto durante el 2005; además se analiza experimentalmente y cuantitativamente el proceso. Los principales obstáculos fueron fallas de diseño en las estructuras de almacenaje, procedimientos inadecuados, contradicción entre lo que se planeaba hacer y lo que realmente se hacía, rotulación confusa y rechazo por parte del personal universitario debido a conflicto de intereses económicos, laborales y de percepción personal de estética. Ese año evaluado se envió a reciclar un total de $53560 \mathrm{~kg}$ (media: 6 kg/persona). Predominaron papel y cartón ( $82 \%$ de los residuos), seguido de vidrio (8\%), plástico (4\%) y metales (2\%) (no se consideró a los residuos orgánicos debido a problemas logísticos). La Oficina de Publicaciones fue la que más residuos produjo y hubo una tendencia a que con el tiempo aumentara la contribución extrauniversitaria (vecinos, funcionarios y estudiantes que traían residuos de sus casas). La ubicación correcta de los residuos mejoró tras un cambio en la rotulación de los contendores problemáticos (Papel 96-95\%, No reciclables $88-90 \%$ y Varios que fue cambiado a Envases -para plásticos, vidrio y aluminio- de 51 a 75\%). La mejoría en la separación de los residuos se debe probablemente al trabajo de educación y a mejoras en la organización y rotulación. A mediados del periodo los residuos enviados al relleno sanitario tuvieron un promedio diario de $606,7 \mathrm{~kg}$, pero solo el $3,6 \%$ era material reciclable, principalmente plásticos y papel. Los sitios en donde se almacenaba el material no reciclable para envío al relleno tuvieron menos higiene cuando las barreras contra vertebrados dificultaban su limpieza. Al diseñar este tipo de proyectos es necesario considerar a todos los actores sociales para evitar conflictos por funciones laborales y entradas económicas. El beneficio de este tipo de proyecto debe verse desde una perspectiva mas amplia, ya que dependiendo de las condiciones de cada país es posible que no se produzca ingreso económico real.

PALABRAS CLAVE

Implementación de reciclaje, ecocampus, gestión ambiental, gestión de residuos, residuos ordinarios. 
En América Latina la producción de residuos es uno de los principales problemas ambientales (PNUMA 2000). Además, la producción de residuos aumentó a una velocidad mayor que la población, así de 1970 al 2000 la producción de desechos pasó de 0,2-0,5kg a 0,5-1,0kg per cápita por día (PNUMA 2000). Costa Rica no escapa a esta tendencia: para el 2005 se reportó la generación de $1.036 \mathrm{~kg}$ per cápita por día (Gaviria \& Soto 2007). Entre los factores que se han mencionado como agravantes de la situación se tienen: desinterés político del gobierno y de las municipalidades por establecer soluciones viables, mala educación ambiental de la población y aumento en los recursos consumidos per cápita (González 2008).

Para aliviar el problema, hay dos estrategias básicas: consumir menos (evitando el desperdicio) y reciclar. Recientemente se han enfocado los esfuerzos también en una producción mas limpia y eficiente, buscando sustitutos menos contaminantes (González 2008) y en el modelo de Emisión Cero de la Universidad de las Naciones Unidas, en el que los consumidores envían al productor los materiales cuando pierden su utilidad (Kuehr 2007). Sin embargo, la aplicación de estos métodos es difícil y aun en países desarrollados el manejo tan complejo ha causado que cada gobierno local desarrolle sus propios protocolos para la recolección, transporte y tratamiento de los residuos sólidos ordinarios, haciendo necesaria la creación de agencias intergubernamentales para dar una buena gestión (Refe \& Domenech 2007). La situación de los países no industrializados es aun mas compleja, pues además se establece todo un esquema de trabajo informal que puede crear conflictos (Corella 2006). Una estrategia actual es el establecimiento de "modelos" que puedan ser analizados, mejorados $y$, posteriormente, generalizados a nivel nacional, como en Curitiba, Brasil (Hülstrunk 2008).

En Costa Rica existen varias iniciativas de los sectores público y privado que buscan establecer modelos de gestión integral viables (Castro 2008); algunas de estas iniciativas cuentan con el apoyo de países mas avanzados en la gestión de residuos sólidos (Hülstrunk 2008). Actualmente, el "Plan de Residuos Sólidos Costa Rica" (conocido como PRESOL), parece ser uno de los mejores esfuerzos que se han realizado en Costa Rica para integrar actores y sectores sociales (Navarro 2008).

Dentro de estos esfuerzos es importante recordar el papel de las universidades como formadoras de los profesionales (Armijo de Vega et al. 2003), pues el establecimiento de sistemas de gestión de residuos adecuados permite que los estudiantes aprendan a través de la participación (Newman 2007, Barrientos et al. 2009). Además, debido a su carácter académico, suelen ser las primeras instituciones públicas en poner en práctica este tipo de iniciativas
(Universidad de Alcalá s.f., Benayas et al. 2002) y en buscar avances en los modelos y diseños establecidos. Por otra parte, las universidades cuentan con el apoyo y presión de una serie de declaraciones internacionales (como son la Declaración de Estocolmo de 1972 y la Declaración de Taillores 1990) que hacen hincapié en la importancia de que las universidades promuevan este tipo de iniciativas (Wright 2002, Armijo de Vega et al. 2003); de esta forma es que han surgido alrededor del mundo universidades que se denominan ecocampus o campus sostenibles. Desafortunadamente, la información que se encuentra disponible sobre estos programas universitarios suele ser cualitativa o informativa. La información cuantitativa es poca, por lo que es difícil comparar el éxito de los programas (Shriberg 2002) y su impacto en la formación ambiental de la comunidad.

A pesar de la importancia de implementar programas de gestión ambiental en las universidades, en Costa Rica no se ha hecho un esfuerzo conjunto del CONARE, que enlaza a las universidades públicas. En el caso de la Universidad Nacional fue hasta finales del 2001 que un proyecto de la escuela de Química de la UNA logró llamar la atención de las autoridades universitarias y culminó en mayo del 2003 con la aprobación de la Política Ambiental (Consejo Universitario de la Universidad Nacional 2003, Sánchez et al. 2004, Barrientos et al. 2009). Fue un proceso participativo de los principales cuerpos colegiados universitarios y de representantes de sindicatos y federaciones estudiantiles. A raíz de esto, en el 2004 se creó el Sistema Institucional de Gestión Ambiental (SIGA-UNA), que manejaba varios proyectos de gestión ambiental (Barrientos et al. 2009). Entre ellos tenía el "Proyecto de Capacitación en Manejo de Desechos Ordinarios". De esta manera la Universidad Nacional se convirtió en la primera universidad estatal costarricense en oficializar a ese nivel los compromisos ambientales que toda institución pública debe respetar. En el 2007 el programa fue reestructurado y actualmente se llama UNA Campus Sostenible, pero mantiene los mismos principios y propósitos del SIGA-UNA (Barrientos et al. 2009).

En este artículo explicamos el funcionamiento del "Proyecto de Capacitación y Manejo de Desechos Ordinarios" y las lecciones aprendidas, con base en una investigación científica formal que utilizó muestreos de los residuos producidos, encuestas al personal y un análisis administrativo crítico de obstáculos para la realización de los objetivos del proyecto durante el 2005.

\section{MATERIALES Y MÉTODOS}

El trabajo se realizó en los campus universitarios Omar Dengo y Presbítero Benjamín Núñez de la Universidad Nacional, Heredia, Costa Rica. Los datos recolectados forman 
parte del trabajo realizado por el Sistema Institucional de Gestión Ambiental (SIGA) en su proyecto "Gestión y Capacitación en Manejo de Desechos Ordinarios" de octubre del 2004 a diciembre del 2005. EI SIGA fue reestructurado y re-inaugurado en el 2007 con el nombre de "UNA Campus Sostenible" (Barrientos et al. 2009). La comunidad universitaria que frecuentó el área de estudio fue de 8000 estudiantes y 1000 funcionarios.

Para la recolección de datos se contó con seis operarios debidamente capacitados en el manejo y selección de residuos sólidos ordinarios, un camión para la recolección de residuos y una balanza con capacidad de $2000 \mathrm{~kg}$ y precisión de 0,5kg.

En este trabajo se evaluó el protocolo oficial y funcionamiento general de recolección de residuos sólidos ordinarios en la Universidad Nacional, además, se analizó: la rotulación utilizada, la constitución de los residuos, la fuente de origen, la calidad de la selección de los residuos en la fuente de origen y la higiene de los lugares de almacenamiento temporal de los residuos destinados al relleno sanitario.

\section{Funcionamiento general del programa}

Entre noviembre del 2004 y diciembre de 2005 se realizaron entrevistas abiertas orales a 120 funcionarios (conserjes, personal administrativo y académico) y a 500 estudiantes. También se hicieron evaluaciones mediante observación directa, con una duración de 40 horas semanales de trabajo de campo, por 50 semanas, para un total de 2000 horas. En todos los casos, el objetivo de las entrevistas y observaciones fue identificar los obstáculos para el buen manejo de residuos sólidos de la universidad.

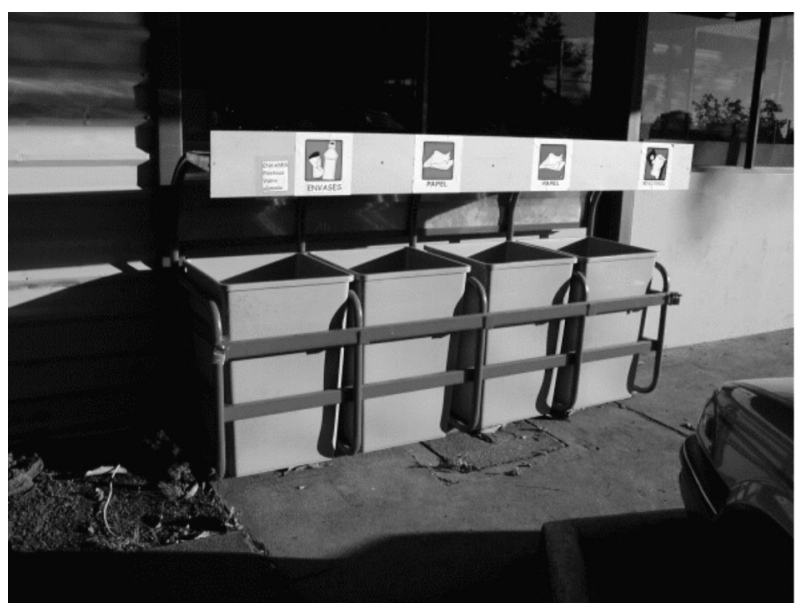

FIG. 1. Batería primaria.
A continuación se describe de manera general el protocolo oficial de manejo de residuos sólidos ordinarios de la Universidad Nacional. Para la recolección del material se partió del postulado de que la mejor gestión de los residuos se da cuando la separación se hace desde la fuente generadora. Con este fin se diseñaron dos tipos de estructura para el depósito de residuos ordinarios denominados recolectores primarios y recolectores secundarios. Los recolectores primarios son estructuras metálicas de $219 \mathrm{~cm}$ de largo por $125 \mathrm{~cm}$ de alto y $63 \mathrm{~cm}$ de ancho (Fig. 1), el diseño incluía el uso de candados para evitar el robo de la estructura y/o de su contenido. Se instalaron 46 recolectores primarios, nueve de ellos en las residencias estudiantiles, y los demás en pasillos y entradas de instalaciones universitarias que contaran con suficiente espacio y estuvieran resguardados de la lluvia. Los recolectores secundarios son estructuras mas grandes tipo jaula con candados para evitar el robo del material (Fig. 2). Se ubicaron nueve recolectores secundarios en lugares que estuvieran cercanos a la calle, pero que a la vez contaran con protección para la lluvia.

Cada recolector primario tiene cuatro contenedores plásticos a los que se les pone bolsas plásticas para que los residuos puedan ser manipulados con mas facilidad e higiene. Las bolsas plásticas que se utilizan son transparentes con el objetivo de facilitar el manejo y clasificación del material en el Centro de Acopio Institucional que es el lugar en el que se completa la separación y se embala el material que será enviado a reciclar. Además, esas bolsas son gruesas para garantizar su reutilización en al menos 5 ocasiones, pero el costo de las mismas es mas elevado que el de las bolsas negras tradicionales. Las bolsas utilizadas en los contenedores primarios son marcadas con el nombre de la unidad administrativa de donde proceden,

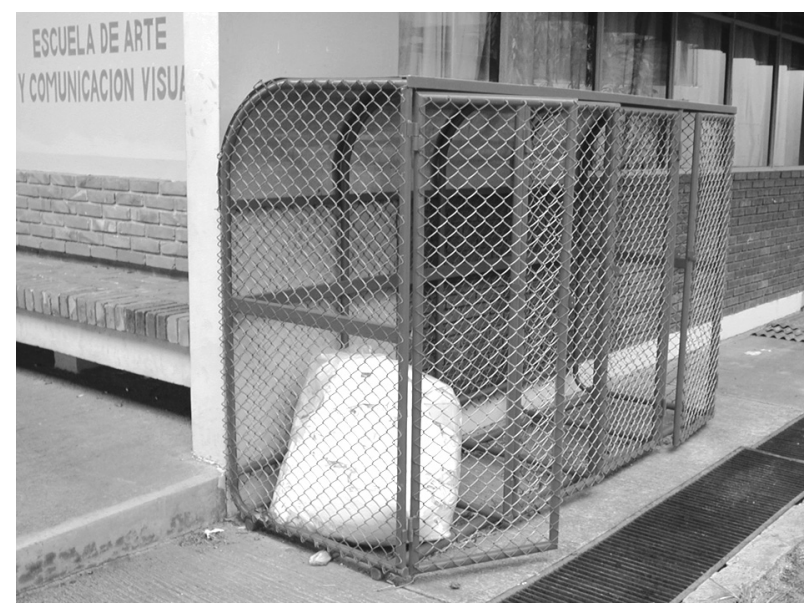

FIG. 2. Contenedor secundario. 
con el fin de llevar un control del origen de los residuos y para que las bolsas sean reutilizadas por la unidad administrativa que las compró. Los contenedores fueron marcados con rótulos adhesivos que tienen un nombre y una imagen representativos del tipo de material que se espera recolectar (Fig. 3). Los residuos se clasificaron originalmente en:

- "Papel", en esta categoría se espera recolectar papel de color, impreso, blanco, periódico, de directorio, cartoncillo y cartón, pero a la vez se espera que se excluyan servilletas y papel higiénico

- "Varios", en la que se espera recolectar plástico, vidrio y metales

- "Orgánico", en esta categoría se espera recolectar alimentos

- "No Recuperable", en la que se espera recolectar todo el material que no puede ser reciclado y cuyo destino final es el relleno sanitario.

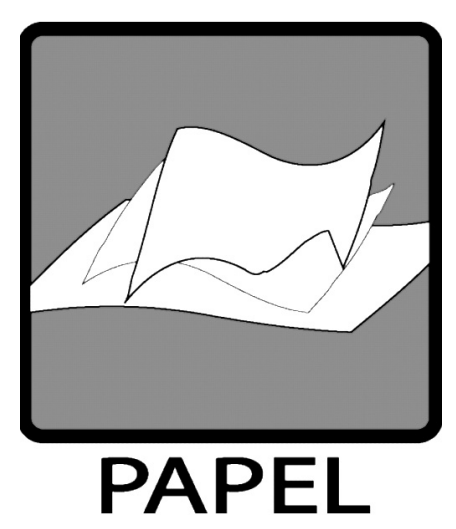

FIG. 3. Ejemplo de los rótulos adhesivos utilizados para ayudarle al productor del desecho a clasificar el material que iba a botar.

Para que la comunidad universitaria aprendiera a seleccionar correctamente los residuos y para que conocieran el funcionamiento del proyecto, se colocaron afiches explicativos en varios sitios de la universidad. En ellos se dan ejemplos de cuál material debe ser depositado en cada contenedor. Además se capacitó presencialmente a mas de 80 funcionarios (incluyendo al $90 \%$ de los conserjes de la institución) y a mas de 300 estudiantes (incluyendo a un $50 \%$ de los estudiantes de las residencias estudiantiles de la universidad).

El protocolo de trabajo aprobado por la universidad indica que el conserje de cada lugar debe recolectar de forma separada los residuos de las oficinas y aulas y llevarlos al contenedor primario mas cercano a su área de trabajo. El protocolo no establece cómo se debe hacer la recolección de residuos sólidos ordinarios en las oficinas y aulas universitarias. Una vez al día el conserje debe cambiar las bolsas de todos los contenedores de los recolectores primarios y llevar el material reciclable y no reciclable al recolector secundario. Según el protocolo el personal del proyecto debe, únicamente, visitar los recolectores secundarios para llevar el material al Centro de Acopio Institucional, recolectar el material "No recuperable" y llevarlo al relleno sanitario.

\section{Evaluación de la rotulación de contenedores}

Con el fin de evaluar el efecto del rótulo de los contenedores primarios en la correcta separación de los residuos por parte de la comunidad universitaria, se realizaron dos tratamientos en los que se analizó el porcentaje del peso del material correctamente depositado. Para el diseño de los tratamientos se consideró las observaciones cualitativas realizadas en las primeras semanas de iniciado el proyecto en las que se encontró que el material depositado en los contenedores rotulados con "Varios" y "No Recuperables" parecían tener un porcentaje de error en la separación mayor que en el rotulado con "Papel".

En un primer tratamiento se utilizaron los nombres que se habían designado originalmente en el proyecto y en un segundo tratamiento se sustituyó "Varios" por "Envases" para botellas plásticas, envases de vidrio y de aluminio (y restos de hierro y cobre) y se agregó al rótulo una lista de residuos que debían ser depositados en ese contenedor. Para los residuos que no pueden ser reciclados como envases tetrabrik, polvo y servilletas, se sustituyó el rótulo "No Recuperable" por "No Reciclable". Como control se mantuvo en ambos tratamientos el rótulo "Papel" para papel, libros, periódicos y cartón. Para cada uno de los tratamientos se analizaron 10 bolsas de cada uno de los 3 tipos de rótulos.

\section{Caracterización de los residuos reciclables generados}

Durante 11 meses (enero a noviembre de 2005) se pesó y clasificó el material reciclable acopiado por el proyecto. Se consideró el peso, tipo de material (papel, plástico, vidrio, hierro, cobre y plomo) y fuente de origen (aproximadamente 80 dependencias universitarias, en el caso del material traído por funcionarios, estudiantes y vecinos de sus hogares se consideró que la fuente era extrauniversitaria). También se analizó el comportamiento mensual de la fuente que mas residuos reciclables produjeron y del material de origen extrauniversitario. 


\section{Evaluación de la separación de los residuos por los productores}

Durante la semana del 2 al 6 de mayo del 2005 se realizó un estudio sobre la cantidad total de residuos ordinarios generados en el campus Omar Dengo en el que se analizó: cantidad y peso de las bolsas utilizadas para enviar el material no recuperable al relleno sanitario; peso de los residuos depositados por los productores en la categoría "No Recuperables"; peso de los residuos depositados por los productores en la categoría "Envases"; peso del material no recuperable depositado en "Envases" y peso de material reciclable depositado en "No Recuperables". El material reciclable que fue depositado en "No Recuperables" se dividió en residuos plásticos (incluyó botellas y bolsas plásticas) y de papel (incluyó cartón y todo tipo de papel reciclable).

\section{Higiene de los lugares de almacenamiento temporal de residuos ordinarios no reciclables}

Durante 5 días en mayo del 2005 se clasificó la higiene de los lugares en donde se depositan las bolsas mientras pasa el camión recolector que lleva los residuos al relleno sanitario. Los sitios se dividieron en dos tipos: 22 lugares protegidos contra perros y otros mamíferos (recolectores secundarios, casitas y canastas elevadas) y 18 lugares desprotegidos (suelo a la orilla de las calles) (Fig. 4). La evaluación de los sitios se hizo de manera cualitativa en: buena (no había residuos fuera de las bolsas ni desprendían mal olor), regular (había pocos residuos fuera de bolsas y no olía mal), mala (había muchos residuos fuera de las bolsas y olía mal) y pésima (había muchos residuos acumulados que se extendían a varios metros alrededor del lugar y olía muy mal).

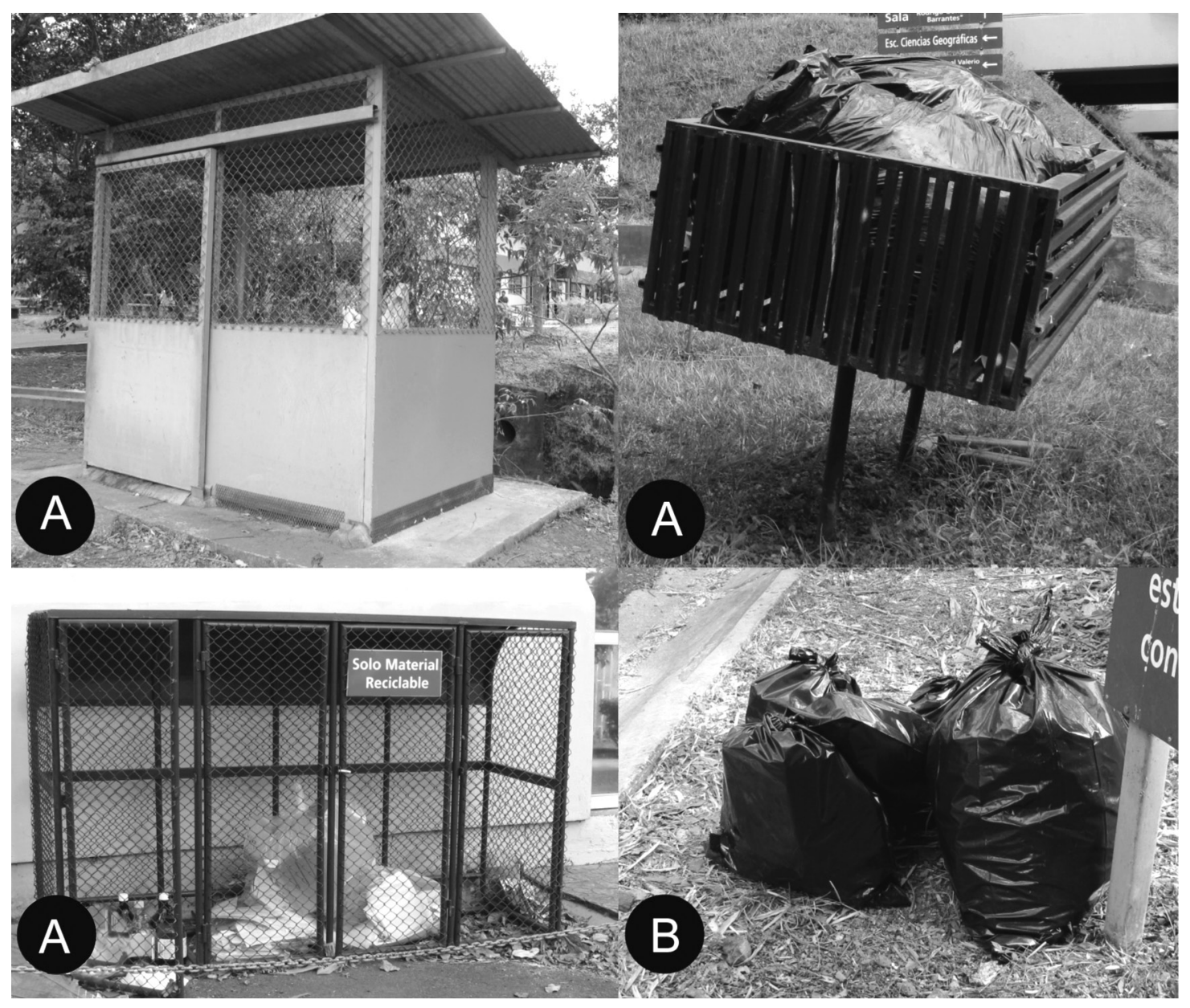

FIG. 4. Estructuras o lugares en los que se pone el material mientras espera ser llevado al relleno sanitario por el servicio de recolección de basura. (A) Lugares protegidos (Casita, canasta, y recolectores secundarios); (B) Lugares desprotegidos (suelo). 


\section{RESULTADOS}

\section{Funcionamiento general del programa}

Se logró identificar cuatro tipos de problema que afectan el desempeño: mal diseño de estructuras recolectoras, procedimientos inadecuados, rotulación confusa, rechazo al programa por razones personales, y rotación de personal y comunidad estudiantil.

\section{a) Mal diseño de estructuras recolectoras}

1. El diseño de los recolectores primarios es inadecuado. El mantenimiento diario de los recolectores primarios es poco práctico debido a que la estructura cuenta con dos candados y un travesaño que hay que quitar para poder cambiar las bolsas de los contenedores. La tabla frontal del recolector primario y en el que se ubican los rótulos adhesivos, dificulta la extracción de las bolsas de los contenedores, especialmente si se han llenado mucho. Durante el periodo de estudio, en varias ocasiones las estructuras frontales se dañaron al tratar de sacar las bolsas. Además, el tamaño de los contenedores es muy grande por lo que las bolsas que contienen papel a veces pesan mas de lo que el funcionario puede manipular con seguridad (máximo para mujeres e $15 \mathrm{~kg}$ y para hombres $20 \mathrm{~kg}$ ).

2. Los recolectores secundarios también presentan problemas de diseño, pues las puertas son muy estrechas y las bolsas se rompen al meterlas y sacarlas de la estructura. Por otra parte, para cubrir adecuadamente las necesidades de la universidad se necesita aumentar la cantidad de estas estructuras.

\section{b) Procedimientos inadecuados}

1. Existe inexactitud en el protocolo pues no se establece cómo se debe hacer la separación de residuos en las oficinas y aulas. Los conserjes han recurrido a diferentes estrategias para solventar esa deficiencia: en las aulas algunos conserjes han instalado un juego de tres basureros en las aulas y pasillos, otros han eliminado los basureros de las aulas y pasillos y otros se quejan de que los residuos que se producen en las aulas y oficinas son depositados en un solo basurero y que ellos deben separar el material para depositarlo en los contenedores.

2. Hay contradicciones entre lo establecido en el protocolo y lo que realmente se hace. No se cumple con el protocolo de actividades y responsabilidades de conserjes y personal del proyecto que dicta la universidad. A diferencia de lo establecido, el material reciclable es recolectado en cada oficina por personal del proyecto. Esto ocurre entre una y dos veces por semana o cuando el personal de la oficina lo solicita; así, los recolectores primarios no cumplen su función, además, con frecuencia el material reciclable se almacena en lugares inapropiados como baños, salas de reuniones y oficinas, causando malestar a los usuarios. Por otra parte, las bolsas de los contenedores de los recolectores primarios no se cambian diariamente y algunos conserjes se niegan a dar el mantenimiento que necesitan estas estructuras, por lo que el trabajo debe ser asumido en la práctica por el personal del proyecto.

3. Aunque el protocolo establece que el proyecto se debe hacer cargo de la recolección del material "No Recuperable", esa función la realiza una compañía externa que se contrata para ese fin. Debido a esa contradicción entre el protocolo y la práctica se instalaron insuficientes recolectores secundarios y se eliminaron algunas estructuras de tipo canasta elevada o "casita" (Fig. 4) en las que se acostumbra acopiar los residuos mientras la compañía encargada los recogía para llevarlos a relleno sanitario. Ello ha provocado que algunos recolectores secundarios se utilicen solo para almacenar material reciclable y otros para almacenar tanto residuos reciclables como residuos no recuperables que deben ser llevados al relleno sanitario. El resultado: confusión e inconformidad entre los funcionarios.

4. La utilización de bolsas transparentes gruesas en los contenedores para material "No Recuperable" no es recomendable ya que su costo es alto y no pueden ser reutilizadas (se envían al relleno sanitario).

5. La asignación de un contenedor del recolector primario para material "No Recuperable" y otro para "Orgánico" no es conveniente. La cantidad de residuos orgánicos que se genera en los pasillos y oficinas es muy poca y el tamaño de los contenedores es muy grande. Además, por la incomodidad para sacar las bolsas de los contenedores primarios, las bolsas no se cambian diariamente, así que se generan problemas de malos olores y plagas.

\section{c) Rotulación confusa}

Pese a las capacitaciones y los afiches, los contenedores rotulados con los términos "Varios" y "No Recuperables" contenían material mal clasificado. Se encontró que los usuarios no establecían la diferencia entre el rótulo "Varios"y el de "No Recuperables". Además los rótulos no eran suficientemente explicativos, pues consistían solo del nombre y un dibujo muy esquemático. 


\section{d) Rechazo del proyecto por razones personales}

El gremio universitario que mostró mayor rechazo al proyecto fue el personal de conserjería. Aproximadamente unos 20 conserjes de 80 se mostraban abiertamente negativos hacia las actividades desarrolladas por el proyecto, otros 10 las apoyaban con entusiasmo y el resto del personal no expresaba ni rechazo ni aceptación de una forma abierta y clara, pero mediante las entrevistas se pudo conocer las razones de su inconformidad.

Durante varios años previos al establecimiento del proyecto de "Gestión y Capacitación en Manejo de Desechos Ordinarios" algunos funcionarios de conserjería vendían el papel de desecho, a veces como un ingreso extra para la oficina donde trabajaban, pero en general esto constituía un ingreso personal, que perdieron al aparecer el proyecto. En general esos conserjes eran quienes más abiertamente rechazaban el proyecto .

La mayor razón de inconformidad de los conserjes que no mostraban una posición clara hacia el proyecto fue la asignación de funciones. Dentro del protocolo del proyecto los contenedores primarios no sustituían a los basureros en los pasillos, oficinas y aulas, por lo que su mantenimiento era visto como un recargo de funciones. Aunque la universidad aprobó el protocolo del proyecto, no se modificó el perfil de funciones de los conserjes. Por otra parte, la comunidad universitaria y los vecinos de la universidad (comunidad extrauniversitaria) comenzaron a traer residuos reciclables de sus casas. Este hecho aumentaba el volumen del material recolectado en los recolectores primarios y los conserjes consideraban que no les correspondían encargarse de ese material. También se mostraron inconformes por la incomodidad para darle mantenimiento a los recolectores primarios (candados, mal olor, peso excesivo de las bolsas y un diseño que dificultaba la extracción de las bolsas).

Otros funcionarios universitarios mostraron rechazo hacia el proyecto por que los recolectores primarios les parecían desagradables estéticamente: rechazaban que estuvieran en lugares visibles. Un caso ilustrativo de esta situación fue la negativa de los funcionarios a que se ubicara una de esas estructuras en el edificio de la rectoría de la universidad. También causaba inconformidad el hecho de que las bolsas plásticas fueran compradas por las unidades administrativas que las usaban, pues eran caras y además el personal del proyecto no devolvía con prontitud las bolsas para ser reutilizadas, lo que los obligaba a comprar una mayor cantidad de bolsas. Otra de las razones de inconformidad por parte del personal administrativo era la incomodidad de tener material de reciclaje guardado en lugares inconvenientes (baños, bodegas, escritorios, etc.).

\section{e) Rotación de funcionarios y estudiantes}

Todos los años se van de la universidad una cantidad considerable de estudiantes y algunos funcionarios, e ingresa un grupo nuevo de estudiantes y funcionarios, que requieren capacitación en el manejo de residuos ordinarios. Además, a pesar de los esfuerzos por capacitar al personal y a los estudiantes, la cantidad de personas que se capacitó fue poca en relación al total de la comunidad universitaria.

\section{Evaluación de la rotulación de contenedores}

El cambio de nombre en los rótulos de "No Recuperables" por "No Reciclables" y de "Varios" por "Envases" (mas una lista de materiales) permitió clasificar mejor los residuos (Cuadro 1). Se encontró que el porcentaje más alto de acierto es para "Papel" con alrededor de 95\% en ambos tratamientos. El mayor aumento en el porcentaje de acierto se produjo con el cambio del rótulo de "Varios" por "Envases" (mas una lista), mejorando el acierto en casi un $25 \%$ (Cuadro 1). El porcentaje de acierto para los rótulos "No Recuperable" y "No Reciclable" fue bastante alto y similar.

Aunque el nombre de "envases" es poco preciso, pues los usuarios podrían depositar envases no reciclables

CUADRO 1

Porcentaje del peso del material correctamente depositado en los contenedores primarios según el rótulo.

\begin{tabular}{lccc}
\hline $\begin{array}{c}\text { Tipo de material } \\
\text { que se esperaba que } \\
\text { depositaran los usuarios }\end{array}$ & $\begin{array}{c}\text { Papel, libros, periódicos } \\
\text { y cartón: \% residuos } \\
\text { correctamente clasificados }\end{array}$ & $\begin{array}{c}\text { Tetrabriks, servilletas y } \\
\text { polvo: \% residuos residuos } \\
\text { correctamente clasificados }\end{array}$ & $\begin{array}{c}\text { Plásticos, vidrio, hierro, aluminio } \\
\text { y cobre: \% residuos residuos } \\
\text { correctamente clasificados }\end{array}$ \\
\hline Rótulos originales & $96 \%$ "Papel" & $88 \%$ "No Recuperable" & $51 \%$ "Varios" \\
Rótulos alternativos & $95 \%$ "Papel" & $90 \%$ "No Reciclable" & $75 \%$ "Envases"
\end{tabular}

Entre comillas el nombre de los rótulos. 
(como son los tetrabrik) y no depositar material reciclable (como varillas de hierro), se consiguió un incremento en el porcentaje de material correctamente depositado.

\section{Caracterización de los residuos reciclables generados.}

Durante un año (2005) el proyecto procesó un total de $53560 \mathrm{~kg}$ de residuos que fueron enviados a reciclar en vez de ir al relleno sanitario y se obtuvo un ingreso de 5 516 dólares (Cuadro 2). La mayor parte de los residuos reciclables que se generaron pertenecían a la categoría “Papel" con un $81,64 \%$, los demás tipos de material reciclable representaron un porcentaje muy bajo, siendo el vidrio el segundo mas importante con tan solo un 8,35 \% del total de peso procesado. El mayor ingreso económico se obtuvo también por el papel, seguido muy lejanamente por el aluminio y el vidrio (Cuadro 2).

\section{CUADRO 2}

Ingreso económico (en dólares), peso $(\mathrm{kg})$ y tipo de material reciclable procesado durante 13 meses de desarrollo del proyecto

\begin{tabular}{lrrr}
\hline $\begin{array}{l}\text { Tipo de material } \\
\text { reciclable }\end{array}$ & Peso $(\mathbf{k g})$ & $\%$ de peso & $\begin{array}{r}\text { Ingreso } \\
\text { económico } \\
\text { (dólares) }\end{array}$ \\
\hline $\begin{array}{l}\text { Papel (varios tipos } \\
\text { y cartón) }\end{array}$ & 43728 & 81,64 & 4168,00 \\
Vidrio & 4470 & 8,35 & 132,00 \\
Plástico & & & \\
(varios tipos) & 2220 & 4,14 & 456,00 \\
Hierro & 2068 & 3,86 & 134,00 \\
Aluminio & 642 & 1,20 & 573,00 \\
Plomo & 395 & 0,74 & 21,00 \\
Cobre & 37 & 0,07 & 32,00 \\
Total & 53560 & 100 & $\mathbf{5 5 1 6 , 0 0}$ \\
& & &
\end{tabular}

La Oficina de Publicaciones de la universidad fue la entidad universitaria que más residuos reciclables generó durante los 11 meses de muestreo, su aporte en relación con otras entidades universitarias se mantuvo relativamente estable a lo largo del tiempo (Spearman, $\mathrm{r}=-0,545$, $\mathrm{n}=11, \mathrm{p}=0,5455)$, alrededor de los $636 \mathrm{~kg}$, lo cual constituye $20,7 \%$ de los residuos reciclables de la universidad (Cuadro 3). El segundo lugar fue ocupado por diversas oficinas y su aporte mensual fue de $8,3 \%$ del material reciclable (Cuadro 3). La cantidad mensual de material reciclable que se recolectaba proveniente de entidades extrauniversitarias (vecinos, funcionarios y estudiantes que traían el material de sus casas) mostró una tendencia a aumentar, aunque esta no fue estadísticamente significativa (Spearman, $r=0,6 ; n=11 ; p=0,0578$ ) (Cuadro3).

\section{Evaluación de la separación de los residuos por los productores.}

Se encontró que en promedio diariamente se envían $606,7 \mathrm{~kg}$ de residuos diarios al relleno sanitario (d.e.= $114,16 ; n=5)$. Con ese fin se utilizaban diariamente un promedio de 112.4 bolsas (d.e. $=11,26 ; n=5$ ), con un promedio de $5.4 \mathrm{~kg}$ por bolsa. El material reciclable clasificado por los usuarios en la categoría "Envases" representó un promedio diario de $139,0 \mathrm{~kg}$ (d.e.=93,8; $\mathrm{n}=5$ ). Dentro del material destinado clasificado como "No Recuperable" por los productores se pudo recuperar un promedio diario de $21,6 \mathrm{~kg}$ (d.e. $=12,16 ; n=5$ ). Ese material mal depositado representó un 3,6\% del total de residuos destinados al relleno sanitario y un 13,5\% del material de la categoría "Envases" producido por la universidad. Dentro del material mal clasificado sólo se encontraron residuos plásticos y de papel los cuales tuvieron igual representación (W- MannWhitney, $W=8 ; p=0.4)$.

\section{Higiene de los lugares de almacenamiento temporal de residuos ordinarios no reciclables.}

Los lugares donde se almacenan los residuos para ser enviados al relleno sanitario generalmente están ubicados a la orilla de la calle. Se encontró que en el $67,5 \%$ de los 40 puntos muestreados la higiene era buena $\left(X^{2}=759\right.$, g.l. $=3, p=0$ ). También se encontró que la higiene del lugar dependía de si existía o no algún tipo de protección contra perros y otros animales $\left(X^{2}=13,61\right.$, g.l. $\left.=3, p=0,0035\right)$, siendo mayor la probabilidad de que la higiene fuera mala si existía protección.

\section{DISCUSIÓN}

Es muy difícil hacer una comparación del desempeño del proyecto de manejo de residuos sólidos ordinarios de la Universidad Nacional, Costa Rica, con otras universidades nacionales y extranjeras debido que casi no se han publicado datos y protocolos de trabajo. Además, por lo general, cada universidad desarrolla sus propias iniciativas en gestión ambiental, las cuales suelen ser muy diferentes unas de otras (Armijo de Vega et al. 2003). El proceso se dificulta también por la falta de índices y porque no siempre se brindan todos los parámetros necesarios para evaluar los resultados. Acquatella (2002) hace hincapié en la necesidad de desarrollar índices económicos adecuados para 


\section{CUADRO 3}

Comportamiento mensual de la cantidad de material de desecho enviado a reciclaje por la oficina de publicaciones (fuente que más residuos reciclables envió), por la dependencia que ocupó el segundo lugar y por fuentes extrauniversitarias.

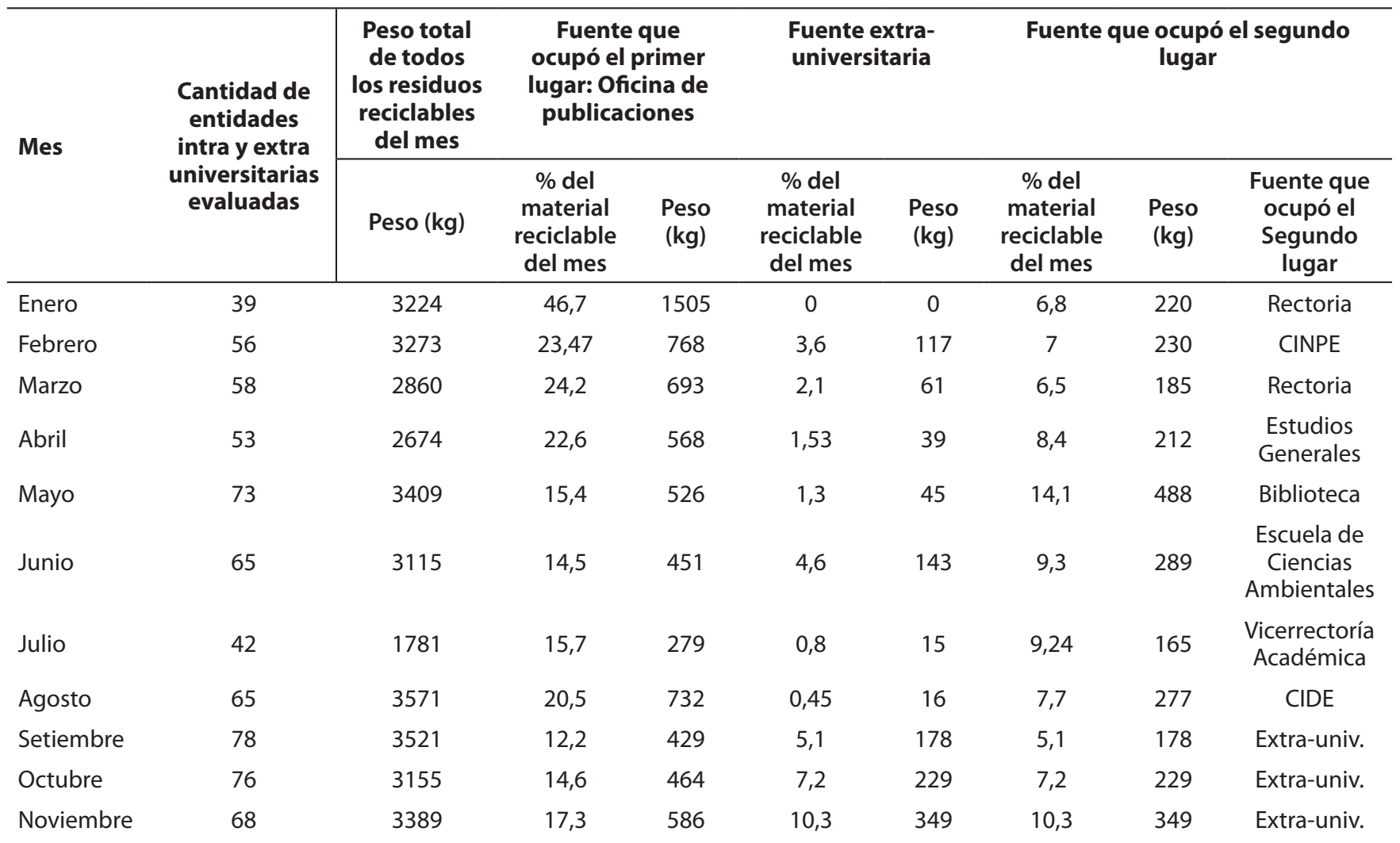

la comparación y evaluación de los procesos de gestión ambiental a nivel macro, pero también es necesario elaborar índices ambientales, económicos y sociales a nivel micro, adecuados para comparar avances y diferencias con otros modelos universitarios. Además es importante fortalecer la investigación experimental en este campo, de manera que las mejoras en los protocolos de trabajo se hagan con base en datos técnicos y no solo en opiniones personales.

Entre los principales problemas que se han reportado para este tipo de proyectos se tiene: la falta de colaboración de la comunidad para separar los residuos que producían (Maldonado 2006), los conflictos de intereses con el transportista que llevaba el material al relleno sanitario (Maldonado 2006) y conflictos de intereses entre el personal administrativo y académico, entre el personal del proyecto de reciclaje y el personal de limpieza y con las personas que antes del proyecto vendían el material reciclable a titulo personal (Armijo de Vega et al. 2003). Todos estos resultados coinciden con los hallados en la Universidad Nacional de Costa Rica. Los conflictos también fueron importantes en este trabajo, por eso se recomienda que el protocolo de trabajo para proyectos de este tipo se planteé de manera que los procedimientos y responsabilidades sean claros y que se vigile que realmente coincidan con lo que se hace. Además, el protocolo debe abarcar todos los procesos desde que se genera el desecho hasta su disposición final, evitando especialmente la confrontación con el personal de conserjería y administrativo de la universidad.

Los resultados de este estudio confirman la reconfiguración de la distribución de beneficios producidos por el manejo de desechos sólidos ordinarios, resultado que también fue informado por Maldonado (2006), por lo que se recomienda que cuando se establezca un proyecto similar se analicen previamente los conflictos de interés y 
los procesos informales que se hayan establecido alrededor de la actividad. Las soluciones a estos conflictos varían según los actores involucrados, en el caso de la Universidad Nacional se recomienda que sea el proyecto de manejo de residuos sólidos quien cubra con los costos de los recipientes, contenedores y bolsas para la separación del material reciclable en toda la universidad y se establezcan lineamientos claros para los funcionarios involucrados en la actividad. El Instituto Tecnológico de Costa Rica detectó esta situación al inicio del establecimiento de su proyecto de reciclaje de residuos. En esa institución se reconoció que el personal de limpieza ya estaba realizando la venta de papel para reciclaje, así que crearon una asociación de ese personal universitario y se le destinó el $65 \%$ del ingreso por ventas de material de reciclaje y el $35 \%$ restante se destino a mejoras del proyecto (Romero et al. 2008). A cambio de ese porcentaje el personal de limpieza tiene la responsabilidad de recoger y trasladar el material de reciclaje. No se tiene información sobre los resultados de esa medida, pero parece una figura interesante que posiblemente logre resolver parte de los conflictos que se generan cuando ya hay procesos informales establecidos.

Al igual que en este proyecto, en otras universidades se ha encontrado que el hecho de tener personal laborando exclusivamente para el proyecto de reciclaje contribuyó al éxito obtenido (Goya-García 2001), por lo que se sugiere que en el diseño de este tipo de proyectos se mantenga esta característica. A diferencia de lo realizado en la Universidad Nacional de Costa Rica, en otras universidades se han colocado no solo los recipientes grandes en pasillos y entradas de edificios, sino que también se colocaron recipientes mas pequeños en las oficinas con resultados positivos (Goya-García 2001). Para mejorar el protocolo se recomienda que en las aulas, pasillos y oficinas universitarias se coloquen recipientes de tamaño pequeño para los residuos reciclables y no reciclables. Para evitar los conflictos laborales con los encargados de limpieza deberían ser ellos los encargados de trasladar el contenido de los contenedores pequeños a los contenedores grandes o primarios como se denominaron en este proyecto. Esos contenedores grandes deberían ser de uso exclusivo para material reciclable, deberían estar a cargo del personal del proyecto de reciclaje y debería mejorarse el diseño para facilitar su manipulación y limpieza. En el mercado existen otros diseños mas prácticos e individualizados que podrían ser considerados. Un ejemplo son los recipientes utilizados en el Instituto Tecnológico de Costa Rica que son de tamaños menores, no cuentan con tantas medidas de seguridad contra robos y por lo tanto son mas fáciles de manipular (Romero et al. 2008). Aunque en el ITCR se utilizaron varios tipos de contenedores no se ha realizado un estudio que permita determinar si hay alguna preferencia de los usuarios por alguno de ellos. Sería muy interesante formular un proyecto que ayude a establecer si el tipo de contenedores afecta la adecuada separación de desechos por parte de los productores y si hay una preferencia en el uso de un tipo determinado de contenedores.

El material no recuperable debería ser recolectado por los conserjes y deben ser ellos quienes llevan el material a los lugares previamente establecidos para que el servicio de recolección de basura los junte y lleve al relleno sanitario. Por otra parte la universidad debe asegurarse de que los desechos no recuperables sean realmente depositados en un relleno sanitario. Ese control es fácil de realizar mediante los comprobantes de pago que emiten los rellenos sanitarios. Es muy curioso que en este trabajo se encontrara que, aunque en general la higiene es buena en los sitios a orillas de la calle en donde se depositan los residuos no reciclables en espera del camión recolector que los lleva al relleno sanitario, tiende a empeorar cuando se cuenta con alguna estructura protectora. Estas estructuras se diseñaron para que los perros, gatos, mapaches y pizotes no las rompan (El mercurio de Calama 2008), pero, posiblemente causen problemas la incomodidad para darles mantenimiento y los conflictos entre el personal universitario en cuanto a quien debe hacerse responsable de su limpieza. Se recomienda que en los protocolos quede bien establecidos que la limpieza de esos sitios corresponde a los conserjes o encargados de limpieza.

Los contenedores secundarios deberían eliminarse debido a su mal diseño y a que, como demuestra este estudio, se mantiene mas limpieza cuando no hay una estructura para depositar este tipo de residuos. El material no recuperable debe ser llevado al relleno sanitario por una compañía externa a la universidad, como se ha hecho hasta la fecha. De esta forma no se contamina el camión de transporte, se evita la confusión entre la comunidad universitaria, en las funciones del personal y rutas de trabajo y se puede concentrar el personal en la capacitación de la comunidad universitaria.

En la rotulación de los contenedores de material reciclable debe utilizar términos mas internacionales y que sean mas descriptivos de lo que se espera recolectar en cada contenedor (p.e. cambiar "Varios" por "Envases"). En el caso del Instituto Tecnológico de Costa Rica se optó por tener cinco recipientes (aluminio, plástico, vidrio, papel y basura), en vez de los tres utilizados en la UNA, sin embargo, en esa institución no se han realizado estudios que demuestren el grado de eficiencia de esa separación y rotulación, solo se indica que es necesario tener estudiantes en el centro de acopio que corrijan los errores de clasificación de los productores de los desechos (Romero et al. 2008). Para el caso de la Universidad Nacional se 
recomienda que los rótulos sean mas demostrativos y se elaboren interactivamente con la participación de estudiantes y funcionarios universitarios, de forma que se integren al proceso de educación ambiental que se desea generar (Escalona \& Pérez 2006, Sammalisto \& Brorson 2008). Contrario a lo que han encontrado otros investigadores (Maldonado 2006), se obtuvo aquí un gran éxito en la separación de residuos por parte de los productores o generadores. Ese éxito mejoró con el transcurso del tiempo, pues aunque en un inicio el $12 \%$ del material que se enviaba al relleno sanitario era reciclable para mediados del periodo evaluado se redujo a solo el 3.6\%. Es posible que esto se deba no solo a la mejora en la rotulación y la capacitación de la comunidad universitaria, sino también al alto nivel de educación ambiental que se ha logrado en Costa Rica en todos los niveles sociales y educativos, pues desde un inicio el porcentaje de éxito fue alto.

En este trabajo se encontró que la cantidad de material orgánico que se genera en las aulas, pasillos y oficinas de la universidad es muy poca para poder ser considerada en un proyecto de reciclaje. No obstante, es recomendable realizar un estudio para identificar, evaluar y caracterizar los residuos orgánicos generados en los comedores universitarios, la finca y los jardines. En otras instituciones de educación superior se ha informado que los residuos orgánicos pueden representar entre un 28 y un $48 \%$ del total (Goya-García 2001, Maldonado 2006), es muy probable que si en un futuro estudio se incluyen los desechos orgánicos producidos en la Universidad Nacional su aporte sea similar al informado por Goya-García (2001) y por Maldonado (2006).

Para mantener una buena "ambientalización" de la universidad y la incorporación de toda la comunidad en el proyecto, debe desarrollarse una estrategia para capacitar a los estudiantes y funcionarios en el momento en que ingresan a la universidad. Además, es importante desarrollar modelos que evalúen la efectividad de la capacitación que recibe la comunidad universitaria en general, pues como se ha visto en otras universidades puede tenerse una mayor ambientalización junto a una menor conciencia ambiental (Benayas et al. 2002). El estudiantado de educación puede estar muy abierto a que la educación ambiental sea vista como un proceso pedagógico en que se puede combinar al gobierno con las comunidades y formar valores y actitudes hacia el ambiente y la sociedad (Escalona \& Pérez 2006). Según nuestros resultados, la educación ambiental debe integrarse en distintos niveles educacionales y sociales: por eso deberían mejorarse los diseños curriculares de todas las carreras que se imparten en la universidad para que incorporen esos valores ambientales y sociales.
Aunque no fue posible encontrar universidades que hubieran hecho un análisis de sus residuos de forma similar a la que se utilizó en este estudio, fue posible modificar los datos de la caracterización de residuos sólidos de tres centros de educación superior (la Universidad de Santiago de Compostela, España, el Centro de Investigación y de Estudios Avanzados del Instituto Politécnico Nacional IPN, en Mérida, México, y el Instituto Tecnológico de Costa Rica) para establecer categorías similares a las usadas en este proyecto. Se obtuvo que también en las otras tres universidades fue el papel el residuo mas importante con un $67 \%, 48 \%$ y $88.5 \%$ respectivamente, en tanto que para la Universidad Nacional se obtuvo un $82 \%$. Los estudios de la universidad española y de la mexicana solo incluyeron una pequeña sección. En el caso del Instituto Tecnológico de Costa Rica no se especifica si incluye toda la universidad o solo una sección. Este estudio consideró la totalidad del campus el cual incluye una editorial conocida como Oficina de Publicaciones y que fue la instancia universitaria que mas residuos reciclables generó durante todo el estudio. Es posible que la nueva tecnología digital hará que disminuya el porcentaje de papel de desecho que generan las imprentas universitarias: la universidad debería establecer una política que apoye la producción de libros digitales.

El porcentaje de plásticos, vidrio y metal obtenidos en la Universidad de Barcelona (10\%, 15\% y $8 \%$ respectivamente) y en el IPN (19\%, 12\% y 5\% respectivamente) fueron mayores que en la Universidad Nacional (4\%, 8\% y 6\%, respectivamente) y en el Instituto Tecnológico de Costa Rica (1,6\%, 9,5\% y 0,4\%, respectivamente) (Goya García 2001, Maldonado 2006, Romero et al. 2008). Ello puede ser reflejo de la diferencia entre países con alto y mediano poder adquisitivo (Merken \& Litten 2007), pues hay que recordar que entre mayor sea el nivel económico de un país, mayor es la tendencia al consumismo y uso de innovaciones tecnológicas y utensilios desechables.

En otros estudios se ha encontrado que el establecimiento de un proyecto como este trae beneficios económicos para la institución (Maldonado 2006). En el caso de la Universidad Nacional no es así, ya que el aporte económico por la venta de material es menor que los egresos debidos a salarios, materiales y equipo. Además el aporte de este proyecto al ambiente es relativamente poco, si se considera que en realidad gran parte de los residuos de papel ya era enviados a reciclar de manera informal por el personal de la universidad.

El beneficio que aporta el proyecto debe verse en la educación ambiental que recibe la comunidad universitaria; en un mayor conocimiento de los residuos que se producen en la universidad (Runfola \& Gallardo 2009); en 
el formar parte de un proyecto integral; en la imagen de la universidad en cuanto a su responsabilidad y valores ambientales; en el tener insumos para mejorar los currículos universitarios (Agamuthu \& Hansen 2007); en reducción de costos ambientales, económicos y sociales para el país (Poltera \& Soto 2008); y en la posibilidad de desarrollar proyectos de investigación interdisciplinarios (Agamuthu \& Hansen 2007). Tales proyectos permiten desarrollar algoritmos y protocolos que posteriormente se puedan aplicar a universos mas complejos, como la adaptación del algoritmo del Sistema de Colonias de Hormigas utilizado para la recolección de residuos sólidos en Atenas, Grecia (Karadimas \& Papatzelou 2007). Un ejemplo de ese beneficio se vio en nuestro caso en el aumento paulatino y constante de la cantidad de material reciclable que vecinos, funcionarios y estudiantes traían de sus hogares.

\section{AGRADECIMIENTOS}

Un especial agradecimiento a Nazira González y Virginia Sánchez (fundadoras del proyecto SIGA-UNA y gestoras de la política ambiental) por el apoyo y la información brindada. La revisión de la separación de los residuos se hizo con el apoyo de María Eugenia Mena y Maribel Hernández (ambas funcionarias del SIGA-UNA). También colaboraron con esta investigación: Manuel Calderón, Flor Orozco, Diego Zúñiga, Gustavo Reyes, Alejandro Montero y Alonso Arrieta (todos personal del SIGA-UNA). La búsqueda de literatura y ayuda asistencial estuvo a cargo de Maribel Zúñiga (UNED, Costa Rica). Se agradece a la Vicerrectoría de la UNED por el apoyo brindado.

\section{REFERENCIAS}

Acquatella, J. 2002. Desafíos y propuestas para la implementación más efectiva de instrumentos económicos en la gestión ambiental de América Latina y el Caribe. (http://www.cepal.org/cgi-bin/getProd.asp?xml=/publicaciones/xml/8/11048/P11048.xml\&xsl=/dmaah/ tpl/p9f.xsl\&base=/tpl/imprimir.xsl; consultado 27 de setiembre, 2006).

Agamuthu, P. \& J. A. Hansen. 2007. Universities in capacity building in sustainable development: focus on solid waste management and technology. Waste management \& research 25: 241-246. (http://eprints.um.edu.my/25/1/Agamuthu.pdf; consultado 20 mayo, 2010).

Armijo de Vega, C., S. Ojeda-Benítez \& M.E. Ramírez-Barreto. 2003. Mexican educational institutions and waste Management programs: a University case study. Resources, conservation and recycling 39: 283-296. (http://red-academica.net/carmijo/wp-content/uploads/2007/01/mexican-educational.pdf; consultado 20 mayo, 2010).
Barrientos, Z., H. Johnson \& M.L. Moreno. 2009. Gestión ambiental en universidades públicas costarricenses: el ejemplo de "UNA-Campus sostenible". Revista Posgrado y Sociedad 9: 81-124. (http://www.uned.ac.cr/SEP/recursos/ revista/documents/UNACampusSostenibleBarrientosJohnson-Moreno.pdf; consultado el 30 de enero, 2010).

Benayas, J., D. Alba \& S. Sánchez. 2002. La ambientalización de los campus universitarios: El caso de la Universidad Autónoma de Madrid. Ecosistemas 11 (3). (http://www.revistaecosistemas.net/pdfs/266.pdf; consultado 20 mayo, 2010).

Castro, R. 2008. Marco legal moderno para la gestión integral de residuos en Costa Rica. Ambientico 178: 7-8. (http:// www.ambientico.una.ac.cr/178.pdf; consultado 20 de mayo, 2010).

Consejo Universitario de la Universidad Nacional. 2003. SCU820-2003. Política Ambiental de la Universidad Nacional. Artículo Tercero, Inciso V de la sesión ordinaria del 22 de mayo, Número 2472. Gaceta No. 7-2003 del 30 de junio del 2003.

Corella, R. 2006. "Buzos" encuentran aguinaldo entre basura de fin de año. La Nación, San José, Costa Rica, Dic 26. (http:// wvw.nacion.com/ln_ee/2006/diciembre/28/pais942519. html; consultado 20 mayo, 2010).

Elmercurio de Calama. 2008. La basuraylos perros. El Mercurio deCalama, Chile. Jun 22. (http://www.mercuriocalama.cl/prontus4_ nots/antialone.html?page=http://www.mercuriocalama.cl/ prontus4_nots/site/artic/20080622/pags/20080622000738. html; consultado el 10 de marzo, 2010).

Escalona, J. \& M. Pérez. 2006. La educación ambiental en la Universidad de los Andes: Un estudio desde la perspectiva de los estudiantes de educación. Educare 10: 483-490. (http://redalyc.uaemex.mx/redalyc/pdf/356/35603411. pdf; consultado 20 mayo, 2010).

Gaviria, L. \& S. Soto. 2007. Situación de la gestión de residuos sólidos en las Municipalidades en Costa Rica: recolección, disposición y recuperación. Tecnología en Marcha 20: 3-7. (http://www.tec.cr/sitios/Vicerrectoria/vie/editorial_tecnologica/Revista_Tecnologia_Marcha/pdf/tecnologia_marcha_20-4/20-4\%203-7.pdf; consultado 20 mayo, 2010).

González, S. 2008. Qué se ha hecho y qué hay que hacer en gestión de residuos sólidos y reciclaje en Costa Rica. Ambientico 176: 14-15. (http://www.ambientico.una.ac.cr/176. pdf; consultado 20 mayo, 2010).

Goya-García, M. 2001. La ambientalización de la Universidad. Un estudio sobre la formación ambiental de los estudiantes de la Universidad de Santiago de Compostela y la Política Ambiental de la Institución. Tesis doctoral. Universidad de Santiago de Compostela. (http://descargas.cervantesvirtual.com/servlet/SirveObras/128105220 26706051321435/005343_4.pdf; consultado el 30 de enero, 2010).

Hülstrunk, W. 2008. ¿Qué es el programa de competitividad y medio ambiente? Ambientico 178: 3-4. (http://www.ambientico.una.ac.cr/178.pdf; consultado 20 mayo, 2010) 
Karadimas, N.V. \& K. Papatzelou. 2007. Optimal solid waste collection routes identified by the ant colony system algorithm. Waste management \& research 25: 139-147. (http:// ntua.academia.edu/documents/0010/7308/_2007-01_ Optimal_Solid_Waste_Collection_Routes_Identified_ by_the_Ant_Colony_System_Algorithm.pdf, consultado 20 de mayo, 2010).

Kuehr, R. 2007. Towards a sustainable society: United Nations University's Zero Emissions Approach. Journal of Cleaner Production 15: 1198-1204.

Maldonado, L. 2006. Reducción y reciclaje de residuos sólidos urbanos en centros de educación superior: Estudio de caso. Ingenieria 10: 59-68. (http://www.ingenieria.uady. $\mathrm{mx} /$ revista/volumen10/reduccion.pdf; consultado 21 de mayo, 2010).

Merken, J. \& L.H. Litten. 2007. The sustainability challenge. New Directions for Institutional Research 134 (Advancing Sustainability in Higher Education): 7-26. (http://search.ebscohost.com/login.aspx?direct=true $\& \mathrm{db}=\mathrm{ehh} \& \mathrm{AN}=2626$ 2500\&site=ehost-live; consultado 21 de mayo, 2010).

Navarro, L. 2008. El plan de residuos sólidos Costa Rica. Proceso y perspectivas. Ambientico 178: 13-15. (http://www.ambientico.una.ac.cr/178.pdf; consultado 21 de mayo, 2010).

Newman, J. 2007. Service learning as an expression of ethics. The sustainability challenge. New Directions for Institutional Research 142: 17-24. (http://search.ebscohost.com/login. aspx?direct=true $\& \mathrm{db}=$ ehh $\& \mathrm{AN}=33385822 \&$ site=ehostlive; consultado 21 de mayo, 2010).

PNUMA (Programa de Naciones Unidas para el Medio Ambiente). 2000. Informe GEO 2000. PNUMA, México DF. 456 p. (http://www.unep.org/geo2000/english/0091.htm; consultado 28 de enero, 2010).

Poltera, N. \& A. Soto. 2008. ¿Cuánto cuestan los residuos? Ambientico 178: 16-18. (http://www.ambientico.una. ac.cr/178.pdf; consultado 21 de mayo, 2010).

Rifé, M.T. \& L.A. Domènech. 2007. Governing the complexity: Solid waste services in the metropolitan area of Barcelona.
Local government studies 33: 625-629. (http://www. ub.es/graap/Termes_Alerm_LGS.pdf; consultado 21 de mayo, 2010).

Romero, L.G., J.C. Salas \& J. Jiménez . 2008. Manejo de desechos en universidades. Estudio de caso: Instituto Tecnológico de Costa Rica. Tecnología en marcha 21: 33-41. (http:// www.tec.ac.cr/sitios/Vicerrectoria/vie/editorial_tecnologica/Revista_Tecnologia_Marcha/pdf/tecnologia_marcha_21-3/33-41.pdf; consultado 20 de mayo, 2010.)

Runfola, J. \& A. Gallardo. 2009. Análisis comparativo de los diferentes métodos de caracterización de residuos urbanos para su recolección selectiva en comunidades urbanas. II Simposio Iberoamericano de Ingeniería de Residuos. Barranquilla, Colombia, 24 y 25 de setiembre de 2009. (http://www.uninorte.edu.co/divisiones/Ingenierias/IDS/ upload/File/Memorias\%20II-SIIR/2a-Runfola-Venezuela-001.pdf, consultado 15 de febrero, 2010).

Sánchez, V., N. González \& M. Quirós. 2004. Gestión ambiental en Universidad Nacional. Ambientico 124: 3-5.

Sammalisto, K. \&T. Brorson. 2008. Training and communication in the implementation of environmental management systems (ISO 14001): a case study at the University of Gävle, Sweden. Journal of Cleaner Production 16: 299-309.

Shriberg, M.P. 2002. Sustainability in U.S. high education: Organizational factors influencing campus environmental performance and leadership. A dissertation submitted in partial fulfilment of the requirements for the degree of Doctor of Philosophy (Natural Resources and Environment) in The University of Michigan. (http://www. housing.umich.edu/pdfs/housing_sustain_report.pdf; consultado 17 de Julio, 2009).

Universidad de Alcalá. s.f. Ecocampus Alcalá. (http://www.uah. es/universidad/ecocampus/inicio.shtm; consultado 17 de julio, 2009).

Wright, T. (2002). Definitions and frameworks for environmental sustainability in higher education. (http://www.ulsf. org/pdf/Wright_Declarations.pdf; consultado $17 \mathrm{de}$ julio, 2009). 